\title{
The interaction of intuition, logic and practice when using the medium of dynamic mathematics
}

\author{
Yagunova E. B. ${ }^{1,2}$, Korovkin N. V. ${ }^{3}$ \\ ${ }^{1}$ University of Electrical Engineering, St.-Petersburg, Russia \\ ${ }^{2}$ Academic University, centre of nanotechnology, St.-Petersburg, Russia \\ ${ }^{3}$ St.-Petersburg State Polytechnical University, St.-Petersburg, Russia
}

\begin{abstract}
Several criteria of improving the efficiency of learning / teaching are given. The results of using of modern educational technology is discussed by the example of addition of vectors.
\end{abstract}

Keywords: Learning strategies, vectors, TI-Nspire, rotated field, advanced ICT for education.

\section{Introduction}

In the process of learning, especially during the training concerning complex issues, we often teeter on the edge of success and failure: have we managed / have we not managed to teach a certain topic, have the students managed / have not managed to learn a certain topic, interesting / boring, useful / useless, easy / difficult. What kind of result we will get often depends on little things - an example that was given in time, a good analogy, even unexpected jokes. Classic "chalk and board" are not sufficient for successful teaching / learning process anymore. On the one hand, the amount of knowledge available in the world today constantly increases. It is becoming increasingly difficult to learn this amount of knowledge in the time allocated for training. On the other hand, life is becoming more dynamic. There is less and less time for "repetition", and it is becoming less likely that the misunderstood topics will be understood immediately afterwards. Therefore, it is hard to underestimate the importance of improving the efficiency of learning / teaching. We can judge about the improvement of education from [3]

- larger volume of studied material

- deeper understanding of the material

- greater interest in the subject

- $\quad$ study of more complex algorithms

- wider associative field

- less time spent

- $\quad$ students giving intuitively correct answers to simple questions

When studying even the simplest educational topics, careful application of modern means of education can significantly increase the effectiveness of the educational process. We will demonstrate this on the example of addition of vectors.

2. Addition of vectors: classical and modern options of presenting the topic

Within the classic tradition of working with pencil and paper, we have always easily trained or learnt the vector addition. 
Let there be given vectors $\overrightarrow{O X}, \overrightarrow{O Y}$. Draw "by hand", "by eye" a parallelogram limited by these vectors -it does not matter if it is a little bit crooked! Draw its diagonal $\overrightarrow{O Z}$ - and the sum of vectors is found. Any student can deal with that. Having learned to add vectors, it is easy to determine decomposition of vectors in the basis $\left\{\overrightarrow{O X_{0}}, \overrightarrow{O Y}\right\}$ - representation of the vector $\overrightarrow{O Z}$ as a linear combination of basis vectors, ie, in the form of $\overrightarrow{O Z}=\alpha \cdot \overrightarrow{O X}+\beta \cdot \overrightarrow{O Y}$. However if we want to find the coordinates of the vector - numbers $\alpha$ and $\beta$ - pencil and paper are not enough. We would at least require a ruler. Let's start from this point to carry out calculations and plotting using the program TI-NspireCAS.

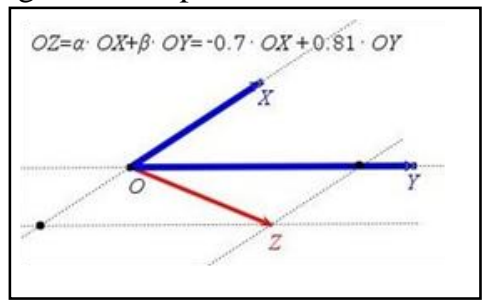

Fig. 1: Interactive decomposition of vector with TI-Nspire.

Interactive drawing allows you to monitor changes of the coordinates of vector $\overrightarrow{O Z}$ when the position of the point $\mathrm{Z}$ changes (Fig. 1). Let's execute several exercises: find the geometrical locus of points $\mathrm{Z}$, such that both coordinates of vector $\overrightarrow{O Z}$ are positive / the coordinates have different signs / both coordinates are negative. A slightly more complex questions: find the geometrical locus of points $\mathrm{Z}$, such that the coordinates of vector $\overrightarrow{\mathrm{OZ}}$ are equal / one coordinate of the vector $\overrightarrow{O Z}$ is twice the value of the other one /is three times the value of the other one / the sum of coordinates is constant. Computer experiments aiming at finding answers to such questions will be very relevant. Using such experiments we can get the an- swer to the above questions. This will create a correct idea of solutions and answers. The only thing that remains is to validate these responses with analytical corroboration. Now that we have mastered the addition of vectors, we will solve three problems. The first of them will give us a powerful tool for solving complex geometric problems. The second will develop mathematical intuition. The third problem will demonstrate the properties of the three-phase current.

\section{Problem 1. Addition of vectors: ap- plication in geometric problems}

Given basis $\left\{\overrightarrow{O X}_{;}, \overrightarrow{O Y}\right\}$. What requirements should the coordinates of the vector $\overrightarrow{O Z}$ meet for the point $\mathrm{Z}$ to be on the line XY? Let's not tell the students a well known answer (the sum of the vector $\overrightarrow{O Z}$ must equal one), and instead offer them to explore that question with the help of a pre-prepared interactive drawing - Fig. 2 (module "Graphs" or module "Geometry"). The program allows to change the position of the point $\mathrm{Z}$ on the line and automatically record the coordinates of vector $\overrightarrow{O Z}$ in the table (module "Lists \& Spreadsheets"). Let's establish the relationship between the coordinates of the vector. In order to do this, we can build a Scatterplot in order to obtain a series of numbers and see that the points lie on the line $\mathrm{y}=1-\mathrm{x}$ (module "Data $\&$ Statistics"). So, we found that the vector $\overrightarrow{O Z}$ represented

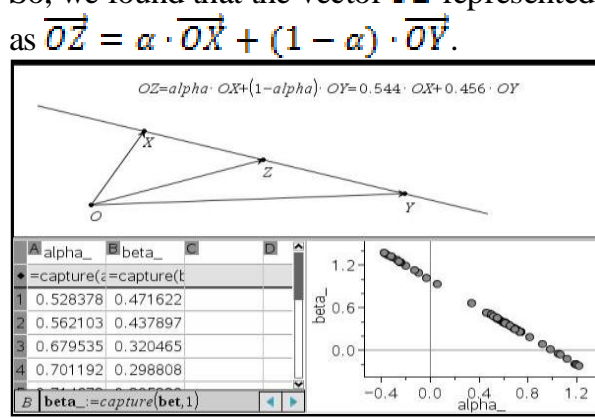

Fig. 2: The equation of line. 
It remains to understand what is the geometric meaning of the number $\alpha$. It is easy to see that when $\alpha=0$, the point $Z$ corresponds with the point $\mathrm{Y}$, and if $\alpha=1-$ it corresponds with the point $\mathrm{X}$. Negative values of the parameter $\alpha$ and values greater than one, correspond to two rays of the line $X Y$. For $0<\alpha<1$, the point $Z$ lies on the segment $X Y$. The final step of our arguments -is finding a relationship between the value of $\alpha$ and the distances between the points $\mathrm{X}, \mathrm{Y}, \mathrm{Z}$. If you want to continue these studies you can answer the same questions for the point $\mathrm{Z}$, when it is not along the line $\mathrm{XY}$, but along any line [4]. Or you can go directly to the applications of the results. While solving this problem we have managed to significantly expand the amount of knowledge on student activities with vectors and vector coordinates in a short time due to the following factors:

- clarity

- diversity of arguments

- active student participation in the development of results.

Having discovered these regularities with the help of interactive drawings, the pupil easily learns vector technology of solving geometric problems. For example, it becomes quite easy to find a ratio in which the segments $\mathrm{AA}$ and $\mathrm{BB}$, joining the vertices $\mathrm{A}$ and $B$ of the triangle $A B C$ with the points on opposite sides, divide each other. Suppose, to make it more definite, $A B^{\prime}: B^{\prime} C=2: 1$, $B A^{3}: A^{3} C=3: 5$. As a basis we take the vectors $\overrightarrow{A B}, \overrightarrow{A C}$. We use the above formulas for the decomposition of the vector in the basis and write the sequence of formulae:

$\overrightarrow{A B^{3}}=\frac{2}{\mathrm{a}} \overrightarrow{A C} ; \overrightarrow{A A^{t}}=\frac{\mathrm{a}}{\mathrm{a}} \overrightarrow{A C}+\frac{5}{\mathrm{a}} \overrightarrow{A B}$.

Let us denote the point of intersection $\overrightarrow{B B^{1}}$ and $\overrightarrow{A A^{t}}$ as $\mathrm{O}$ and determine the vector $\overrightarrow{A O}$ in two ways:

$\overrightarrow{A O}=\beta \cdot \overrightarrow{A A^{t}}=$

$=\alpha \cdot \overrightarrow{A B}+(1-\alpha) \cdot \overrightarrow{A B} \cdot \mathrm{p}$

Using formula (1), we obtain

$\beta \cdot\left(\frac{3}{8} \overrightarrow{A C}+\frac{5}{8} \overrightarrow{A B}\right)=$

$=\alpha \cdot \overrightarrow{A B}+(1-\alpha) \cdot\left(\frac{2}{a} \overrightarrow{A C}\right)$.

and find $\alpha$ and $\beta$ from this system of equations

$\left\{\begin{array}{c}\beta \cdot \frac{a}{g}=(1-\alpha) \cdot \frac{2}{a} \\ \beta \times \frac{5}{g}=\alpha\end{array}\right.$

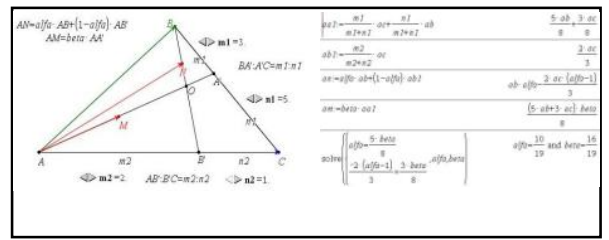

Fig. 3: Illustration and calculation for problem 2.

To execute all these calculations and to find the solution of the system it is most appropriate to use the unit Calculator, so that the 
calculations do not overshadow the content side of what is happening (Fig. 3). We find that $\left\{\begin{array}{l}\alpha=10 / 19 \\ \beta=16 / 19\end{array}\right.$, i.e. $A O: O A^{x}=16: 3, B O: O B^{x}=9: 10$.

Using this handy multifunctional program allows you to master this rather complex algorithm due to:

- accurate drawings of high quality

- visible relationship between the position of vector and its coordinates

- dynamic character of pictures changes of the coordinates of the vector when you change the position of the point

- fast execution of calculations

- opportunity to check the answer on the drawing - "by eye" or by direct measurement.

What we found out while executing this task are the conditions that are met by a vector drawn to a point on the line. Let's verify our geometrical intuition now.

\section{Problem 2. Addition of vectors: checking the accuracy of our geo- metrical intuition}

Let us assume that the point $\mathrm{Z}$ is not moving in a straight line but in a circle. What are the conditions that are met by vector $\overrightarrow{O Z}$ in this situation? This question is rather trivial. It is sufficient to recall that in the case of an orthonormal vector basis, the coordinates of the vector, originating from the zero of coordinate system, will coincide with the Cartesian coordinates of the vector's end. Of course, if the point $\mathrm{Z}$ moves in a circle, its coordinates (i.e. the coordinates of the vector) satisfy the equation of the circle. This means that the scatterplot of coefficients $\alpha$ and $\beta(\overrightarrow{O Z}=\alpha \cdot \overrightarrow{O X}+\beta \cdot \overrightarrow{O Y})$ in the case of an orthonormal basis $\{\overrightarrow{O X} ; \overrightarrow{O Y}\}$ will represent a circle. This result is obvious, but only for a person with advanced mathematical intuition and rich associative field (and their development is one of our ultimate goals). The school pupil would find the very formulation of the problem far from trivial. The task is multi-layered: it is necessary to develop a meaningful approach to the vector basis, understanding of the concept of vector coordinates, understanding of the functional relationship (relationship of coordinates). The resulting circle is unexpected for the student, and it motivates him to further mathematical experiments. How will the relationship between the coefficients of the change in the position or the radius of the circle along which the point $Z$ ? How will the relationship between the coefficients change in case of the change of basis vectors? Ask the student to predict the response and check its accuracy with experiment. After we have trained the intuition, let us move on to the third problem.

\section{Problem 3. Addition of vectors: the properties of three-phase current.}

Resolution of this problem (found, in particular, in 1889 by the Russian scientist M.O.Dolivo-Dobrovolsky) has changed our world in a degree not smaller than the invention of the computer. Consider the three vectors $-\overrightarrow{e_{1}}, \overrightarrow{e_{2}}$ and $\overrightarrow{e_{a}}$, starting from the same point and be offset from each other by an angle $2 \pi / 3$. Let us determine that the lengths of these vectors are not constant but vary with time according to the following rule:

$\left|\overrightarrow{e_{1}}\right|=\mathrm{E} \cos \omega t_{\mathrm{t}}$ 
$\left|\overrightarrow{e_{2}}\right|=\mathrm{E} \cos (\omega t-2 \pi / 3)$,

$\left|\overrightarrow{e_{a}}\right|=E \cos (\omega t+2 \pi / 3)$.

Describe the time dependence of the vector $\operatorname{sum} \vec{e}=\overrightarrow{e_{1}}+\overrightarrow{e_{2}}+\overrightarrow{e_{a}}$. To solve this problem analytically, it is necessary to choose a basis, to record the coordinates of each vector in this basis and perform coordinate wise addition. After the application of a number of trigonometric formulae we obtain the following formal expression

$\arg (\vec{e})=\omega t+\alpha_{0},|\vec{e}|=\frac{\mathrm{aE}}{2} \cdot \cos \left(\alpha_{0}\right)(5)$

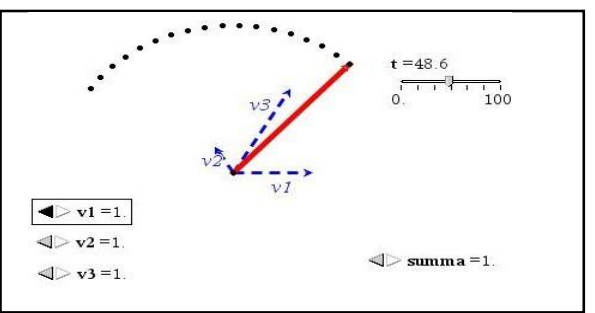

Fig. 4: Rotating vector of resulting field.

This expression is quite cumbersome and its interpretation is difficult. The main conclusion was left in the background, while actions with vectors and their coordinates and trigonometric transformations have moved to forefront of problem solving. But using these formulae, we have proved that the vector $\vec{e}$ rotates counterclockwise with angular velocity $\omega$, without changing its length. Basically, we have just reproduced the contents of the most important topic of electrical engineering - "rotating magnetic field", which serves as grounds for understanding of principles of work of all types of three-phase alternating current electrical machines [1,2]! Got it? Hardly. Thus the students do not understand it either. Even many engineers do not.

Let us reconstruct the process of studying this material. First, we will show students three vectors whose length changes with time -we will show them the dynamics. Now while looking at the interactive drawing, let us ask the students to define these vectors - with coordinates or with length and direction. Equations (4) are now rather obvious. Let us construct the sum of the vectors (and we already know how to do it to perfection), then run the animation. Now all we have to do is look at the screen (Fig. 4) - there is a vector of constant length $\vec{e}$ revolving around the zero of coordinates. One cannot help but be amazed that already in the nineteenth century there were people who were able to imagine this picture by simply looking at the formulae (5).

Once the geometric interpretation of the results is clear, the student can easily understand all of the ideas of constructing a 3-phase (and multiphase) devices offered by M.O.Dolivo-Dobrovolsky. Electric power industry of the nineteenth century was based on direct current. After the discovery of the described method of producing a rotating magnetic field, a new power indus- 
try has appeared - and it still exists all around us now. However these conversations are rather aimed at students of technical universities. And for a school pupil this is just a beautiful problem, which opens a lot of questions for further research. How can we change the rotation of the vector $\vec{e}$ tothe opposite? What would happen if the vectors $\overrightarrow{e_{1}}, \overrightarrow{e_{2}}$ and $\overrightarrow{e_{a}}$ were offset from each other at different angles? Is it possible to get a sum with the same properties if we add up four vectors instead of three?

We have managed to solve a nice geometrical problem with major applications in engineering. The solution is understandable for any student through the use of modern educational technology. The formulae describing the condition and the answer to the problem are now natural and obvious. We have saved time and energy which can be spent on discussion of applications of the result.

\section{References}

[1] K.S.Demirchyan, L.R.Neyman, and N.V. Korovkin, Theory of Electrical Engineering, v.1, v.2, St. Petersburg, Piter, p. 512,p. 430, 2009.[in Russian]

[2] N.V.Korovkin, K.S.Demirchyan, L.R. Neyman and Chechurin V.L. Theory of Electrical Engineering. Wei Zhao,Xi Xiao,Yuxiang Wang,Lizhu Tong,Yi Ma,Biqiu Xu,Jin Xing,Higher Education Press China, Beijing, pp.1136.

[3] S.N. Pozdnyakov, and S.G. Ivanov, Computers in productive teaching of mathematics or how information technologies can support intellectual freedom of the learner. The 10-th International Congress on Mathematical Education, National presentation: Russia, Selected materials, Copenhagen, Denmark, July 4-11, 2004, p. 115-124.

[4] E.B. Yagunova, Radius-vectors: from the "rules of parallelogram" to the solving of geometrical problems. Computer instruments in the school. 2012. N2, 12-21. 